\title{
DOMAINS BOUNDED BY ANALYTIC JORDAN CURVES
}

\author{
BY R. J. SIBNER ${ }^{1}$
}

Communicated by W. Fuchs, August 22, 1969

It is well known that repeated application of the Riemann mapping theorem shows that any finitely connected plane domain is conformally equivalent to a domain whose boundary components are analytic curves. A corresponding result has not previously been shown for arbitrary domains of infinite connectivity-the only cases being those domains for which the Koebe conjecture is known to be true (cf. [2]).

We will show that any domain with countably many boundary components is conformally equivalent to a domain bounded by analytic Jordan curves and points.

1. A limit boundary component of a domain is a boundary component, at least one of whose points is a point of accumulation of points on other boundary components. A weak limit boundary component of a domain is a point limit boundary component which corresponds to a point under every conformal map of the domain. A circle domain is a domain bounded by circles and points.

In a recent paper [2, Theorem 4] the author has obtained a new proof, using quasiconformal mappings, of the following result due to Strebel: If $D$ is a domain with weak limit boundary components, then $D$ is conformally equivalent to a circle domain.

An examination of this proof shows that the assumption that the limit boundary components are point boundary components and that they are weak is used only in the last step-to show that the images of the limit boundary components are points. Consequently, we obtain

Theorem 1. Let $D$ be an arbitrary planar domain. Then $D$ is conformally equivalent to a domain $K$, all of whose isolated boundary components are circles or points.

AMS Subject Classification. Primary 3040.

Key Words and Phrases. Conformal mapping, infinitely connected domains, analytic Jordan curves, Koebe conjecture, circle domains, transfinite induction.

1 Research partially supported by NSF grant GP-8556. 
REMARK. For the proof of our main theorem which follows, a weaker version of the above would suffice-the conclusion being that all isolated boundary components are analytic Jordan curves or points. A proof of this, which is more elementary than the proof indicated above of Theorem 1, will appear in [3].

2. Let $\Gamma^{0}=\Gamma^{0}(D)$ denote the collection of boundary components of the plane domain $D$. We form the sequence $\Gamma^{\alpha}=\Gamma^{\alpha}(D)$ (cf. [1], also [4])

$$
\Gamma^{0} \supseteq \Gamma^{1} \supseteq \cdots \supseteq \Gamma^{\omega} \supseteq \Gamma^{\omega+1} \supseteq \cdots \supseteq \Gamma^{\alpha} \supseteq \cdots
$$

where $\omega$ denotes the ordinal type of the natural numbers and $\alpha$ is an arbitrary ordinal number. If $\alpha$ has a predecessor $\alpha-1$, then $\Gamma^{\alpha}$ is defined as the subset of $\Gamma^{\alpha-1}$ of components which contain points which are limit points of the components in $\Gamma^{\alpha-1}$ so that if $\Gamma^{0}$ is given a metric space structure (see [1]) then $\Gamma^{\alpha}$ is the derived set of $\Gamma^{\alpha-1}$. If $\alpha$ is a limit ordinal, we define $\Gamma^{\alpha}=\bigcap_{\beta<\alpha} \Gamma^{\beta}$.

We recall [1] that if $\Gamma^{0}$ is countable then there exists a (first) ordinal $\eta$ such that $\Gamma^{\eta+1}$ is empty. If $\Gamma^{0}$ is uncountable, then the sequence $\Gamma^{\beta}$ does not terminate. The intersection $\cap \Gamma^{\beta}$ over all $\beta$ is called the perfect kernel of $\Gamma^{0}$. Every component in the perfect kernel is a limit boundary component of other components in the perfect kernel. Note that the "ordinal type" of a boundary component is invariant under conformal maps of the domain.

3. Our main result is

THEOREM 2. Let $D$ be a domain with countably many boundary components. Then $D$ is conformally equivalent to a domain bounded by analytic Jordan curves and points.

A brief outline of the proof, by transfinite induction on $\alpha$, follows. The complete proof will appear in [3].

Consider the following statement: (We use the term "analytic Jordan curves" to include points.)

$S(\alpha)$ : There exist conformal maps $f(\gamma): D \rightarrow D_{\gamma}$ for all $\gamma \leqq \alpha$, and conformal maps $h(\gamma, \delta): D_{\gamma} \rightarrow D_{\delta}$ for $\gamma \leqq \delta \leqq \alpha$ such that (for $\left.\gamma \leqq \delta \leqq \epsilon \leqq \alpha\right)$ :

(i) $h(\delta, \epsilon) \circ h(\gamma, \delta)=h(\gamma, \epsilon)$.

(ii) $h(\gamma, \delta)$ is the restriction of a conformal map of the domain bounded by the elements of $\Gamma^{\gamma}\left(D_{\gamma}\right)$.

(iii) $f(\delta)=h(\gamma, \delta) \circ f(\gamma)$.

(iv) The elements of $\Lambda^{\alpha}\left(D_{\alpha}\right)=\Gamma^{0}\left(D_{\alpha}\right)-\Gamma^{\alpha}\left(D_{\alpha}\right)$ are analytic Jordan curves.

The crucial condition is (iv). For $\alpha=\eta+1$ this says (since $\Gamma^{\eta+1}=0$ ) 
that the domain $D_{\eta+1}$, which is conformally equivalent to $D$, is bounded by analytic Jordan curves.

$S(0)$ is trivially true for $f(0)=h(0,0)=$ identity. Suppose $S(\beta)$ is true for all $\beta<\alpha$. We show the truth of $S(\alpha)$.

Case 1. If $\alpha$ is not a limit ordinal and hence has a predecessor $\alpha-1$, then there exists a conformal map $f(\alpha-1): D \rightarrow D_{\alpha-1}$ such that $\Lambda^{\alpha-1}\left(D_{\alpha-1}\right)$ consists of analytic Jordan curves. Let $h(\gamma, \alpha)$ be the restriction to $D_{\alpha-1}$ of the map obtained by application of Theorem 1 to the domain bounded by the elements of $\Gamma^{\alpha-1}\left(D_{\alpha-1}\right)$. The maps $f(\alpha)$ and $h(\gamma, \alpha)$ for arbitrary $\gamma$ are defined so that conditions (i) and (iii) hold. Using the fact that an analytic Jordan curve, lying in the domain of analyticity of a conformal map, is carried by the map into an analytic Jordan curve, conditions (i)-(iv) for $\alpha$ can be immediately verified.

Case 2. If $\alpha$ is a limit ordinal it is necessary to use a diagonalization procedure and a normal family argument (see [3]).

From the proof of Theorem 2 we obtain the following generalization:

COROLlARY 1. An arbitrary domain (countably or uncountably many boundary components) is conformally equivalent to a domain, all of whose boundary components except those in the perfect kernel are analytic Jordan curves.

We obtain also

COROLLARY 2. If every boundary component of an arbitrary domain $D$ is a nondegenerate continua containing at least one point which is not a point of accumulation of points on other boundary components, then $D$ is conformally equivalent to a domain bounded entirely by nondegenerate analytic Jordan curves. (The hypothesis implies that $D$ has at most countably many boundary components.)

\section{REFERENCES}

1. F. Hausdorff, Mengenlehre, de Gruyter, Berlin, 1935; English transl., Chelsea, New York, 1957. MR 7, 419; MR 19, 111.

2. R. J. Sibner, Remarks on the Koebe Kreisnormierungsproblem, Comment. Math. Helv. 43 (1968), 289-295. MR 37 \#5380.

3. - Uniformizations of infinitely connected domains, Proc. Conf. Riemann Surface Theory (Stony Brook, N. Y., 1969) (to appear).

4. K. Strebel, Über das Kreisnormierungsproblem der konformen Abbildung, Ann. Acad. Sci. Fenn. Ser. A. I. Math.-Phys. No. 101 (1951), 22 pp. MR 14, 549.

Rutgers University, New Brunswick, New Jersey 08903 